\title{
Non-Regenerative Multicarrier MIMO Relay Communications Based on Minimization of Mean-Squared Error
}

\author{
Yue Rong \\ Department of Electrical and Computer Engineering, Curtin University of Technology, Bentley, WA 6102, Australia \\ E-mail: y.rong@curtin.edu.au
}

\begin{abstract}
In this paper we propose non-regenerative multicarrier multiple-input multiple-output (MIMO) relay techniques that minimize the mean-squared error (MSE) of the signal waveform estimation. We establish the closed-form optimal precoding matrices at the source and relay nodes in the absence of the direct source-destination link. Interestingly, we show that the proposed precoding matrices jointly convert the multicarrier MIMO relay channel into parallel single-input single-output (SISO) relay channels. In order to reduce the computational complexity of the optimal algorithm, a suboptimal precoding approach based on an upper-bound of the MSE expression is developed. Numerical examples illustrate a significant performance improvement of the proposed algorithms over the existing techniques.
\end{abstract}

\section{INTRODUCTION}

Recently, cooperative communications have been the subject of renewed research [1]. Both non-regenerative and regenerative cooperative strategies have been developed [1], [2]. In non-regenerative approaches, the relay node only amplifies the received signal and retransmits it. Therefore, the complexity of the non-regenerative approach is much lower than that of the regenerative approach. This advantage is particularly important when all nodes are equipped with multiple antennas, since decoding multiple data streams involves much more computational efforts than decoding single data stream.

Non-regenerative approaches have been proposed to maximize the mutual information of singlecarrier multiple-input multiple-output (MIMO) relay systems [3] and multicarrier MIMO relay systems [4]. However, in practical communication systems, an important objective is to minimize the transmission error which is closely related to the mean-squared error (MSE) of the signal waveform estimation.

In this paper, we propose non-regenerative multicarrier MIMO relay algorithms to minimize the MSE of the signal waveform estimation. We study the case where the direct link between the source and destination nodes is sufficiently weak that can be ignored. We show that the optimal source and relay precoding matrices jointly convert the multicarrier MIMO relay channel into parallel single-input single-output (SISO) relay channels. We develop an alternating algorithm which optimally distributes the available transmission power among these SISO relay channels.

Considering that the computational complexity of the alternating algorithm may be high for practical communication systems, we develop a suboptimal algorithm which uses an upper-bound of the MSE expression as the objective function. This algorithm trades a slight performance degradation for a tremendously reduced computational complexity.

Numerical examples in Section IV illustrate a significant performance improvement of both optimal and suboptimal algorithms compared with the existing techniques.

\section{BACKGROUND}

We consider a three-node multicarrier MIMO communication system where the source node transmits information to the destination node with the aid of one relay node. The source, relay, and destination nodes are equipped with $N_{s}, N_{r}$, and $N_{d}$ antennas, respectively. Due to its merit of simplicity, the nonregenerative strategy is applied at the relay node to process and forward the received signal.

The communication process between the source and destination nodes is completed in two time slots. At the first time slot, the modulated signal sequence at the source node is divided into $N_{c}$ blocks. We denote $N_{b}^{n}, n=1, \cdots, N_{c}$ as the number of symbols in the $n$th sub-block. Then the $N_{b}^{n} \times 1$ vector of signal $\mathbf{s}^{n}(t)$ is linearly precoded which can be written as

$$
\mathbf{x}^{n}(t)=\mathbf{B}^{n} \mathbf{s}^{n}(t), \quad n=1, \cdots, N_{c}
$$

where $\mathbf{B}^{n}$ is an $N_{s} \times N_{b}^{n},\left(N_{s} \geq N_{b}^{n}\right)$ precoding matrix for the $n$th sub-block of the source signal sequence. The precoded vector of signal $\mathbf{x}^{n}(t)$ is transmitted to the relay node via the $n$th subcarrier. The received signal can be written as

$$
\mathbf{y}_{r}^{n}(t)=\mathbf{H}_{s}^{n} \mathbf{x}^{n}(t)+\mathbf{v}_{s}^{n}(t), \quad n=1, \cdots, N_{c}
$$

where $\mathbf{H}_{s}^{n}$ is an $N_{r} \times N_{s}$ MIMO channel matrix between the source and relay nodes, $\mathbf{y}_{r}^{n}(t)$ and $\mathbf{v}_{s}^{n}(t)$ are the received signal and the additive Gaussian noise vectors at the relay node, respectively. Hereafter, the superscript $n$ denotes the corresponding variables for the $n$th subcarrier.

At the second time slot, the source node is silent. While the relay node multiplies (linearly precodes) the received signal vector at the $n$th subcarrier with an $N_{r} \times N_{r}$ matrix $\mathbf{F}^{n}$ and transmits the precoded signal vector $\mathbf{x}_{r}^{n}(t+1)=\mathbf{F}^{n} \mathbf{y}_{r}^{n}(t)$ to the destination node. The received signal vector at the $n$th subcarrier of the destination node can be written as

$$
\begin{aligned}
\mathbf{y}_{d}^{n}(t+1) & =\mathbf{H}_{r}^{n} \mathbf{x}_{r}^{n}(t+1)+\mathbf{v}_{d}^{n}(t+1) \\
& =\mathbf{H}_{r}^{n} \mathbf{F}^{n} \mathbf{H}_{s}^{n} \mathbf{B}^{n} \mathbf{s}^{n}(t)+\mathbf{H}_{r}^{n} \mathbf{F}^{n} \mathbf{v}_{s}^{n}(t)+\mathbf{v}_{d}^{n}(t+1)
\end{aligned}
$$


where $\mathbf{H}_{r}^{n}$ is an $N_{d} \times N_{r}$ MIMO channel matrix between the relay and destination nodes, $\mathbf{y}_{d}^{n}(t+1)$ and $\mathbf{v}_{d}^{n}(t+1)$ are the received signal and the additive Gaussian noise vectors at the destination node, respectively. We assume that $\mathbf{H}_{s}^{n}$ and $\mathbf{H}_{r}^{n}, n=1, \cdots, N_{c}$ are all quasi-static and known by all nodes. Note that if the noise vectors are spatially correlated such that $\mathbf{C}_{v_{s}}^{n} \triangleq \mathrm{E}\left[\mathbf{v}_{s}^{n}(t)\left(\mathbf{v}_{s}^{n}(t)\right)^{H}\right] \neq \mathbf{I}_{N_{r}}$ and/or $\mathbf{C}_{v_{d}}^{n} \triangleq \mathrm{E}\left[\mathbf{v}_{d}^{n}(t)\left(\mathbf{v}_{d}^{n}(t)\right)^{H}\right] \neq \mathbf{I}_{N_{d}}$, pre-whitening of the received signals can be performed at the relay and destination nodes such that

$$
\tilde{\mathbf{y}}_{d}^{n}(t+1)=\tilde{\mathbf{H}}_{r}^{n} \tilde{\mathbf{F}}^{n} \tilde{\mathbf{H}}_{s}^{n} \mathbf{B}^{n} \mathbf{s}^{n}(t)+\tilde{\mathbf{H}}_{r}^{n} \tilde{\mathbf{F}}^{n} \tilde{\mathbf{v}}_{s}^{n}(t)+\tilde{\mathbf{v}}_{d}^{n}(t+1)
$$

where $\mathbf{I}_{n}$ denotes an $n \times n$ identity matrix, $\mathrm{E}[\cdot]$ stands for the statistical expectation, $(\cdot)^{H}$ denotes the matrix Hermitian transpose, and

$$
\begin{array}{ll}
\tilde{\mathbf{H}}_{s}^{n}=\left(\mathbf{C}_{v_{s}}^{n}\right)^{-\frac{1}{2}} \mathbf{H}_{s}^{n}, & \tilde{\mathbf{v}}_{s}^{n}(t)=\left(\mathbf{C}_{v_{s}}^{n}\right)^{-\frac{1}{2}} \mathbf{v}_{s}^{n}(t), \\
\tilde{\mathbf{F}}^{n}=\mathbf{F}^{n}\left(\mathbf{C}_{v_{s}}^{n}\right)^{\frac{1}{2}}, & \tilde{\mathbf{y}}_{d}^{n}(t+1)=\left(\mathbf{C}_{v_{d}}^{n}\right)^{-\frac{1}{2}} \mathbf{y}_{d}^{n}(t+1), \\
\tilde{\mathbf{H}}_{r}^{n}=\left(\mathbf{C}_{v_{d}}^{n}\right)^{-\frac{1}{2}} \mathbf{H}_{r}^{n}, & \tilde{\mathbf{v}}_{d}^{n}(t+1)=\left(\mathbf{C}_{v_{d}}^{n}\right)^{-\frac{1}{2}} \mathbf{v}_{d}^{n}(t+1) .
\end{array}
$$

From (4) we see that all noises are independent and identically distributed (i.i.d.). Thus, in the following, without loss of generality, we assume i.i.d. Gaussian noise with zero mean and unit variance, and use (3) as the system input-output model.

We assume that a linear receiver is used at the destination node to retrieve the transmitted signals at all subcarriers. The estimated signal waveform is given by

$$
\hat{\mathbf{s}}^{n}(t)=\left(\mathbf{W}^{n}\right)^{H} \mathbf{y}_{d}^{n}(t+1), \quad n=1, \cdots, N_{c}
$$

where $\mathbf{W}^{n}$ is an $N_{d} \times N_{b}^{n}$ weight matrix at the $n$th subcarrier.

\section{Minimal MSE Relay Design}

The weight matrix of the optimal linear receiver which minimizes the MSE is given by

$$
\mathbf{W}^{n}=\left[\overline{\mathbf{H}}^{n}\left(\overline{\mathbf{H}}^{n}\right)^{H}+\mathbf{H}_{r}^{n} \mathbf{F}^{n}\left(\mathbf{F}^{n}\right)^{H}\left(\mathbf{H}_{r}^{n}\right)^{H}+\mathbf{I}_{N_{d}}\right]^{-1} \overline{\mathbf{H}}^{n}, \quad \forall n
$$

where we define $\overline{\mathbf{H}}^{n} \triangleq \mathbf{H}_{r}^{n} \mathbf{F}^{n} \mathbf{H}_{s}^{n} \mathbf{B}^{n}$ and assume that $\mathrm{E}\left[\mathbf{s}^{n}(t)\left(\mathbf{s}^{n}(t)\right)^{H}\right]=\mathbf{I}_{N_{b}^{n}}, n=1, \cdots, N_{c}$. The minimal MSE is written as

$$
\begin{aligned}
& \mathrm{MSE}=\sum_{n=1}^{N_{c}}\left[\operatorname { t r } \left(\left[\mathbf{I}_{N_{d}}+\overline{\mathbf{H}}^{n}\left(\overline{\mathbf{H}}^{n}\right)^{H}\right.\right.\right. \\
& \left.\left.\left.\times\left(\mathbf{H}_{r}^{n} \mathbf{F}^{n}\left(\mathbf{F}^{n}\right)^{H}\left(\mathbf{H}_{r}^{n}\right)^{H}+\mathbf{I}_{N_{d}}\right)^{-1}\right]^{-1}\right)+N_{b}^{n}-N_{d}\right]
\end{aligned}
$$

where $\operatorname{tr}(\cdot)$ stands for the matrix trace.

\section{A. Optimal Relay Precoding Matrix}

It can be seen from (5) that MSE is a function of $\mathbf{G}^{n} \triangleq$ $\mathbf{B}^{n}\left(\mathbf{B}^{n}\right)^{H}$ and $\mathbf{F}^{n}, n=1, \cdots, N_{c}$. The optimal $\mathbf{G}^{n}$ and $\mathbf{F}^{n}$ can be found by the following optimization problem

$$
\begin{aligned}
\min _{\left\{\mathbf{G}^{n}\right\},\left\{\mathbf{F}^{n}\right\}} & \text { MSE } \\
\text { s.t. } & \sum_{n=1}^{N_{c}} \operatorname{tr}\left(\mathbf{G}^{n}\right) \leq p_{s} \\
& \sum_{n=1}^{N_{c}} \operatorname{tr}\left(\mathbf{F}^{n}\left[\mathbf{H}_{s}^{n} \mathbf{G}^{n}\left(\mathbf{H}_{s}^{n}\right)^{H}+\mathbf{I}_{N_{r}}\right]\left(\mathbf{F}^{n}\right)^{H}\right) \leq p_{r}
\end{aligned}
$$

where for a matrix $\mathbf{A},\left\{\mathbf{A}^{n}\right\} \triangleq \mathbf{A}^{1}, \mathbf{A}^{2}, \cdots, \mathbf{A}^{N_{c}}$. Here (7) and (8) are constraints for the transmission power at the source and relay nodes, respectively, and $p_{s}>0$ and $p_{r}>0$ are the corresponding available power.

For any given feasible $\mathbf{G}^{n}$, let us define

$$
\mathbf{A}^{n} \triangleq \mathbf{H}_{s}^{n} \mathbf{G}^{n}\left(\mathbf{H}_{s}^{n}\right)^{H}, \quad n=1, \cdots, N_{c}
$$

and expand (5) by using the matrix inversion lemma. We can write MSE at the $n$th subcarrier as

$$
\begin{aligned}
\mathrm{MSE}^{\mathrm{n}}= & N_{b}^{n}-N_{d}+\operatorname{tr}\left(\mathbf{I}_{N_{d}}-\mathbf{H}_{r}^{n} \mathbf{F}^{n} \mathbf{A}^{n}\left(\mathbf{F}^{n}\right)^{H}\left(\mathbf{H}_{r}^{n}\right)^{H}\right. \\
& \left.\times\left[\mathbf{H}_{r}^{n} \mathbf{F}^{n}\left(\mathbf{A}^{n}+\mathbf{I}_{N_{r}}\right)\left(\mathbf{F}^{n}\right)^{H}\left(\mathbf{H}_{r}^{n}\right)^{H}+\mathbf{I}_{N_{d}}\right]^{-1}\right) .
\end{aligned}
$$

From (10) we find that $\mathrm{MSE}^{\mathrm{n}}$ depends only on $\mathbf{F}^{n}$ and $\mathbf{A}^{n}$. Therefore, the optimal structure of $\mathbf{F}^{n}$ and $\mathbf{G}^{n}$ can be obtained for each subcarrier separately.

From (8) and (10) we see that for any given feasible $\mathbf{A}^{n}$, the optimal $\mathbf{F}^{n}$ can be found by solving the following problem

$$
\begin{aligned}
\max _{\mathbf{F}^{n}} \operatorname{tr} & \left(\mathbf{H}_{r}^{n} \mathbf{F}^{n} \mathbf{A}^{n}\left(\mathbf{F}^{n}\right)^{H}\left(\mathbf{H}_{r}^{n}\right)^{H}\right. \\
& \left.\times\left[\mathbf{H}_{r}^{n} \mathbf{F}^{n}\left(\mathbf{A}^{n}+\mathbf{I}_{N_{r}}\right)\left(\mathbf{F}^{n}\right)^{H}\left(\mathbf{H}_{r}^{n}\right)^{H}+\mathbf{I}_{N_{d}}\right]^{-1}\right) \\
\text { s.t. } \operatorname{tr} & \left(\mathbf{F}^{n}\left(\mathbf{A}^{n}+\mathbf{I}_{N_{r}}\right)\left(\mathbf{F}^{n}\right)^{H}\right) \leq p_{r}^{n}
\end{aligned}
$$

where $p_{r}^{n}$ denotes the power allocated to the $n$th subcarrier satisfying

$$
\sum_{n=1}^{N_{c}} p_{r}^{n} \leq p_{r}, \quad p_{r}^{n} \geq 0 .
$$

If we introduce

$$
\mathbf{X}^{n} \triangleq \mathbf{H}_{r}^{n} \mathbf{F}^{n}\left(\mathbf{A}^{n}+\mathbf{I}_{N_{r}}\right)^{\frac{1}{2}}
$$

then (11) can be written as

$$
\begin{aligned}
\operatorname{tr}\left(\mathbf{X}^{n}\left(\mathbf{A}^{n}+\mathbf{I}_{N_{r}}\right)^{-\frac{1}{2}} \mathbf{A}^{n}\left(\mathbf{A}^{n}+\mathbf{I}_{N_{r}}\right)^{-\frac{1}{2}}\right. \\
\left.\times\left(\mathbf{X}^{n}\right)^{H}\left[\mathbf{X}^{n}\left(\mathbf{X}^{n}\right)^{H}+\mathbf{I}_{N_{d}}\right]^{-1}\right) .
\end{aligned}
$$

Let us define

$$
\begin{aligned}
& \mathbf{A}^{n} \triangleq \mathbf{U}_{a}^{n} \boldsymbol{\Lambda}_{a}^{n}\left(\mathbf{U}_{a}^{n}\right)^{H} \\
& \mathbf{X}^{n} \triangleq \mathbf{U}_{x}^{n} \boldsymbol{\Lambda}_{x}^{n}\left(\mathbf{V}_{x}^{n}\right)^{H} \\
& \mathbf{H}_{r}^{n} \triangleq \mathbf{U}_{r}^{n} \boldsymbol{\Lambda}_{r}^{n}\left(\mathbf{V}_{r}^{n}\right)^{H}
\end{aligned}
$$

as the eigendecomposition of $\mathbf{A}^{n}$ and the singularvalue decomposition of $\mathbf{X}^{n}$ and $\mathbf{H}_{r}^{n}$, respectively. Substituting (15)(17) back into (13) and solving (13) for $\mathbf{F}^{n}$, we get

$$
\mathbf{F}^{n}=\mathbf{V}_{r}^{n}\left(\boldsymbol{\Lambda}_{r}^{n}\right)^{+} \mathbf{Q}_{r}^{n} \boldsymbol{\Lambda}_{x}^{n} \mathbf{Q}_{x}^{n}\left(\boldsymbol{\Lambda}_{a}^{n}+\mathbf{I}_{N_{r}}\right)^{-\frac{1}{2}}\left(\mathbf{U}_{a}^{n}\right)^{H}
$$

where $(\cdot)^{+}$denotes matrix pseudo-inverse, and

$$
\mathbf{Q}_{r}^{n} \triangleq\left(\mathbf{U}_{r}^{n}\right)^{H} \mathbf{U}_{x}^{n}, \quad \mathbf{Q}_{x}^{n} \triangleq\left(\mathbf{V}_{x}^{n}\right)^{H} \mathbf{U}_{a}^{n} .
$$

Note that both $\mathbf{Q}_{x}^{n}$ and $\mathbf{Q}_{r}^{n}$ are unitary matrices.

Substituting (15), (16) back into the objective function (14), we have

$\operatorname{tr}\left(\mathbf{Q}_{x}^{n}\left(\boldsymbol{\Lambda}_{a}^{n}+\mathbf{I}_{N_{r}}\right)^{-1} \boldsymbol{\Lambda}_{a}^{n}\left(\mathbf{Q}_{x}^{n}\right)^{H}\left(\boldsymbol{\Lambda}_{x}^{n}\right)^{T}\left[\mathbf{\Lambda}_{x}^{n}\left(\boldsymbol{\Lambda}_{x}^{n}\right)^{T}+\mathbf{I}_{N_{d}}\right]^{-1} \boldsymbol{\Lambda}_{x}^{n}\right)$ 
where $(\cdot)^{T}$ denotes the matrix transpose. Substituting (18) back into (12), we can rewrite the power constraint as

$$
\operatorname{tr}\left(\left(\boldsymbol{\Lambda}_{r}^{n}\right)^{+} \mathbf{Q}_{r}^{n} \boldsymbol{\Lambda}_{x}^{n}\left(\boldsymbol{\Lambda}_{x}^{n}\right)^{T}\left(\mathbf{Q}_{r}^{n}\right)^{H}\left(\left(\boldsymbol{\Lambda}_{r}^{n}\right)^{+}\right)^{T}\right) \leq p_{r}^{n} .
$$

Interestingly, we see from (19) and (20) that the objective function is invariant to $\mathbf{Q}_{r}^{n}$, while $\mathbf{Q}_{x}^{n}$ is irrelevant to the power constraint. Therefore, we can choose $\mathbf{Q}_{x}^{n}$ which maximizes (19), and $\mathbf{Q}_{r}^{n}$ that minimizes the left hand side of (20).

It can be proven using [5] that $\mathbf{Q}_{x}^{n}=\mathbf{D}_{N_{r}}$, or equivalently $\mathbf{V}_{x}^{n}=\mathbf{U}_{a}^{n} \mathbf{D}_{N_{r}}^{H}$ maximizes (19). Here $\mathbf{D}_{m}$ denotes an arbitrary $m \times m$ diagonal matrix with unit norm diagonal elements, i.e., $\left|\left[\mathbf{D}_{m}\right]_{i, i}\right|=1, i=1, \cdots, m$. The maximum of (19) is

$$
\operatorname{tr}\left(\left(\boldsymbol{\Lambda}_{a}^{n}+\mathbf{I}_{N_{r}}\right)^{-1} \boldsymbol{\Lambda}_{a}^{n}\left(\boldsymbol{\Lambda}_{x}^{n}\right)^{T}\left[\boldsymbol{\Lambda}_{x}^{n}\left(\boldsymbol{\Lambda}_{x}^{n}\right)^{T}+\mathbf{I}_{N_{d}}\right]^{-1} \boldsymbol{\Lambda}_{x}^{n}\right)
$$

where the diagonal elements of $\boldsymbol{\Lambda}_{a}^{n}$ and $\boldsymbol{\Lambda}_{x}^{n}$ are arranged in the same order. Similarly, using [5] we can also show that $\mathbf{Q}_{r}^{n}=\mathbf{D}_{N_{d}}$, or equivalently $\mathbf{U}_{x}^{n}=\mathbf{U}_{r}^{n} \mathbf{D}_{N_{d}}$ minimizes the left hand side of (20). The minimum is

$$
\operatorname{tr}\left(\left(\boldsymbol{\Lambda}_{r}^{n}\right)^{+} \boldsymbol{\Lambda}_{x}^{n}\left(\boldsymbol{\Lambda}_{x}^{n}\right)^{T}\left(\left(\boldsymbol{\Lambda}_{r}^{n}\right)^{+}\right)^{T}\right)
$$

where the diagonal elements of $\boldsymbol{\Lambda}_{r}^{n}$ and $\boldsymbol{\Lambda}_{x}^{n}$ are arranged in the same order. For simplicity, we let $\mathbf{U}_{x}^{n}=\mathbf{U}_{r}^{n}$ and $\mathbf{V}_{x}^{n}=\mathbf{U}_{a}^{n}$, then the optimal $\mathbf{F}^{n}$ is written as

$$
\mathbf{F}_{0}^{n}=\mathbf{V}_{r}^{n}\left(\boldsymbol{\Lambda}_{r}^{n}\right)^{+} \boldsymbol{\Lambda}_{x}^{n}\left(\boldsymbol{\Lambda}_{a}^{n}+\mathbf{I}_{N_{r}}\right)^{-\frac{1}{2}}\left(\mathbf{U}_{a}^{n}\right)^{H} .
$$

\section{B. Optimal Source Matrix}

We start to derive the optimal $\mathbf{G}^{n}, n=1, \cdots, N_{c}$. Let us define $\mathbf{H}_{s}^{n} \triangleq \mathbf{U}_{s}^{n} \boldsymbol{\Lambda}_{s}^{n}\left(\mathbf{V}_{s}^{n}\right)^{H}$ as the singularvalue decomposition of $\mathbf{H}_{s}^{n}$. Substituting $\mathbf{H}_{s}^{n}$ back into (9) and solving $\mathbf{G}^{n}$, we get

$$
\mathbf{G}^{n}=\mathbf{V}_{s}^{n}\left(\boldsymbol{\Lambda}_{s}^{n}\right)^{+} \mathbf{Q}_{s}^{n} \boldsymbol{\Lambda}_{a}^{n}\left(\mathbf{Q}_{s}^{n}\right)^{H}\left(\left(\boldsymbol{\Lambda}_{s}^{n}\right)^{T}\right)^{+}\left(\mathbf{V}_{s}^{n}\right)^{H}
$$

where $\mathbf{Q}_{s}^{n} \triangleq\left(\mathbf{U}_{s}^{n}\right)^{H} \mathbf{U}_{a}^{n}$ is a unitary matrix. Thus we have

$$
\operatorname{tr}\left(\mathbf{G}^{n}\right)=\operatorname{tr}\left(\left(\boldsymbol{\Lambda}_{s}^{n}\right)^{+} \mathbf{Q}_{s}^{n} \boldsymbol{\Lambda}_{a}^{n}\left(\mathbf{Q}_{s}^{n}\right)^{H}\left(\left(\boldsymbol{\Lambda}_{s}^{n}\right)^{T}\right)^{+}\right) .
$$

From [5], we know that the minimum of (22) is obtained when $\mathbf{Q}_{s}^{n}=\mathbf{D}_{N_{r}}$, or equivalently $\mathbf{U}_{s}^{n}=\mathbf{U}_{a}^{n} \mathbf{D}_{N_{r}}^{H}$. In other words, among all $\mathbf{G}^{n}$ satisfying (9), the optimal one that minimizes the source node power consumption is

$$
\mathbf{G}_{0}^{n}=\mathbf{V}_{s}^{n}\left(\boldsymbol{\Lambda}_{s}^{n}\right)^{+} \boldsymbol{\Lambda}_{a}^{n}\left(\left(\boldsymbol{\Lambda}_{s}^{n}\right)^{T}\right)^{+}\left(\mathbf{V}_{s}^{n}\right)^{H}
$$

where the diagonal elements of $\boldsymbol{\Lambda}_{s}^{n}$ and $\boldsymbol{\Lambda}_{a}^{n}$ are arranged in the same order.

From (21) and (23) we see that at each subcarrier, the optimal relay matrix $\mathbf{F}_{0}^{n}$ "matches" the right singular vectors of the relay-destination channel $\mathbf{H}_{r}^{n}$, and the left singular vectors of the source-relay channel $\mathbf{H}_{s}^{n}$. While the optimal source matrix $\mathbf{G}_{0}^{n}$ "matches" the right singular vectors of $\mathbf{H}_{s}^{n}$. Therefore, the overall multicarrier MIMO relay channel is decomposed into parallel SISO relay channels. Based on this observation, we can write

$$
\begin{aligned}
\mathbf{F}_{0}^{n} & =\mathbf{V}_{r}^{n} \boldsymbol{\Lambda}_{f}^{n}\left(\mathbf{U}_{s}^{n}\right)^{H} \\
\mathbf{G}_{0}^{n} & =\mathbf{V}_{s}^{n} \boldsymbol{\Lambda}_{g}^{n}\left(\mathbf{V}_{s}^{n}\right)^{H}
\end{aligned}
$$

where $\Lambda_{f}^{n}$ and $\Lambda_{g}^{n}$ are diagonal matrices.

\section{Optimal Joint Source and Relay Power Allocation}

Substituting (24) and (25) back into (5), we can express the optimal MSE as

$$
\begin{aligned}
& \operatorname{MSE}_{0}=\sum_{n=1}^{N_{c}} \operatorname{tr}\left(\left[\mathbf{I}_{N_{b}^{n}}+\boldsymbol{\Lambda}_{g}^{n}\left(\boldsymbol{\Lambda}_{s}^{n}\right)^{T} \boldsymbol{\Lambda}_{f}^{n}\left(\boldsymbol{\Lambda}_{r}^{n}\right)^{T}\right.\right. \\
& \left.\left.\times\left(\boldsymbol{\Lambda}_{r}^{n}\left(\boldsymbol{\Lambda}_{f}^{n}\right)^{2}\left(\boldsymbol{\Lambda}_{r}^{n}\right)^{T}+\mathbf{I}_{N_{d}}\right)^{-1} \boldsymbol{\Lambda}_{r}^{n} \boldsymbol{\Lambda}_{f}^{n} \boldsymbol{\Lambda}_{s}^{n}\right]^{-1}\right) .
\end{aligned}
$$

It can be easily seen from (26) that for an efficient utilization of the transmission power at the source and relay nodes, $N_{b}^{n}$ should satisfy $N_{b}^{n} \leq M \triangleq \min \left(N_{s}, N_{r}, N_{d}\right), \forall n$. Thus the optimal joint source and relay design problem is written as

$$
\begin{aligned}
\min _{\left\{\lambda_{g, i}^{n}\right\},\left\{\lambda_{f, i}^{n}\right\}} & \sum_{n=1}^{N_{c}} \sum_{i=1}^{M}\left(1+\frac{\left(\lambda_{r, i}^{n} \lambda_{f, i}^{n} \lambda_{s, i}^{n}\right)^{2} \lambda_{g, i}^{n}}{\left(\lambda_{r, i}^{n} \lambda_{f, i}^{n}\right)^{2}+1}\right)^{-1} \\
\text { s.t. } & \sum_{n=1}^{N_{c}} \sum_{i=1}^{M} \lambda_{g, i}^{n} \leq p_{s} \\
& \sum_{n=1}^{N_{c}} \sum_{i=1}^{M}\left(\lambda_{f, i}^{n}\right)^{2}\left[\left(\lambda_{s, i}^{n}\right)^{2} \lambda_{g, i}^{n}+1\right] \leq p_{r} \\
& \lambda_{g, i}^{n} \geq 0, \quad \lambda_{f, i}^{n} \geq 0, \quad \forall i, n
\end{aligned}
$$

where $\lambda_{r, i}^{n}, \lambda_{f, i}^{n}, \lambda_{s, i}^{n}, \lambda_{g, i}^{n}$ are the diagonal elements of $\boldsymbol{\Lambda}_{r}^{n}$, $\Lambda_{f}^{n}, \Lambda_{s}^{n}, \Lambda_{g}^{n}$, respectively, and for a scalar $a,\left\{a_{i}^{n}\right\} \triangleq$ $a_{1}^{1}, \cdots, a_{M}^{N_{c}}$. To simplify the notations, we define

$$
\begin{array}{ll}
a_{i}^{n} \triangleq\left(\lambda_{s, i}^{n}\right)^{2}, & b_{i}^{n} \triangleq\left(\lambda_{r, i}^{n}\right)^{2}, \\
x_{i}^{n} \triangleq \lambda_{g, i}^{n}, & y_{i}^{n} \triangleq\left(\lambda_{f, i}^{n}\right)^{2}\left[\left(\lambda_{s, i}^{n}\right)^{2} \lambda_{g, i}^{n}+1\right], \quad \forall i, n .
\end{array}
$$

Problem (27)-(30) can be equivalently converted to the following problem

$$
\begin{aligned}
\min _{\left\{x_{i}^{n}\right\},\left\{y_{i}^{n}\right\}} & \sum_{n=1}^{N_{c}} \sum_{i=1}^{M} \frac{a_{i}^{n} x_{i}^{n}+b_{i}^{n} y_{i}^{n}+1}{a_{i}^{n} b_{i}^{n} x_{i}^{n} y_{i}^{n}+a_{i}^{n} x_{i}^{n}+b_{i}^{n} y_{i}^{n}+1} \\
\text { s.t. } & \sum_{n=1}^{N_{c}} \sum_{i=1}^{M} x_{i}^{n} \leq p_{s}, \quad x_{i}^{n} \geq 0, \quad \forall i, n \\
& \sum_{n=1}^{N_{c}} \sum_{i=1}^{M} y_{i}^{n} \leq p_{r}, \quad y_{i}^{n} \geq 0, \quad \forall i, n .
\end{aligned}
$$

Although each term $\left(a_{i}^{n} x_{i}^{n}+b_{i}^{n} y_{i}^{n}+1\right) /\left(a_{i}^{n} b_{i}^{n} x_{i}^{n} y_{i}^{n}+a_{i}^{n} x_{i}^{n}+\right.$ $\left.b_{i}^{n} y_{i}^{n}+1\right)$ is a quasi-convex function [6] of $x_{i}^{n}$ and $y_{i}^{n}$, we do not know the convexity of the sum of all $M N_{c}$ terms (i.e., the objective function (31)) with respect to $\left\{x_{i}^{n}\right\}$ and $\left\{y_{i}^{n}\right\}$. In the following, we develop an alternating algorithm to find the solution to problem (31)-(33). Note that both the objective and the constraints of this problem are symmetric in $\left\{x_{i}^{n}\right\}$ and $\left\{y_{i}^{n}\right\}$. Therefore, in an alternating fashion, we can update one group of variables by fixing the other group of variables. In particular, with given $\left\{y_{i}^{n}\right\}$, the optimization problem of updating $\left\{x_{i}^{n}\right\}$ can be written as

$$
\begin{aligned}
\min _{\left\{x_{i}^{n}\right\}} & \sum_{n=1}^{N_{c}} \sum_{i=1}^{M} \frac{a_{i}^{n} x_{i}^{n}+\beta_{i}^{n}}{\beta_{i}^{n}\left(a_{i}^{n} x_{i}^{n}+1\right)} \\
\text { s.t. } & \sum_{n=1}^{N_{c}} \sum_{i=1}^{M} x_{i}^{n} \leq p_{s}, \quad x_{i}^{n} \geq 0, \quad \forall i, n
\end{aligned}
$$


where $\beta_{i}^{n} \triangleq b_{i}^{n} y_{i}^{n}+1, \forall i, n$. The solution to problem (34), (35) is the well-known water-filling solution and is given by

$$
x_{i}^{n}=\frac{1}{a_{i}^{n}}\left[\sqrt{\frac{a_{i}^{n}\left(\beta_{i}^{n}-1\right)}{\mu \beta_{i}^{n}}}-1\right]^{\dagger}, \quad \forall i, n
$$

where for a real-valued number $x,[x]^{\dagger} \triangleq \max (x, 0)$, and $\mu>$ 0 is the solution to the following nonlinear equation

$$
\sum_{n=1}^{N_{c}} \sum_{i=1}^{M} \frac{1}{a_{i}^{n}}\left[\sqrt{\frac{a_{i}^{n}\left(\beta_{i}^{n}-1\right)}{\mu \beta_{i}^{n}}}-1\right]^{\dagger}=p_{s} .
$$

The left hand side of (36) is monotonically decreasing with respect to $\mu$. Thus $\mu$ can be found, for example, by the bisection method [6].

Similarly, with given $\left\{x_{i}^{n}\right\}$, we can update $\left\{y_{i}^{n}\right\}$, by solving the following problem

$$
\begin{aligned}
\min _{\left\{y_{i}^{n}\right\}} & \sum_{n=1}^{N_{c}} \sum_{i=1}^{M} \frac{b_{i}^{n} y_{i}^{n}+\gamma_{i}^{n}}{\gamma_{i}^{n}\left(b_{i}^{n} y_{i}^{n}+1\right)} \\
\text { s.t. } & \sum_{n=1}^{N_{c}} \sum_{i=1}^{M} y_{i}^{n} \leq p_{r}, \quad y_{i}^{n} \geq 0, \quad \forall i, n
\end{aligned}
$$

where $\gamma_{i}^{n} \triangleq a_{i}^{n} x_{i}^{n}+1, \forall i, n$. The solution to problem (37), (38) is similar to that of problem (34), (35).

The alternating algorithm solving problem (31)-(33) works in the following steps. First, the algorithm is initialized with a random feasible $\left\{y_{i}^{n}\right\}$. Then the algorithm iteratively updates $\left\{x_{i}^{n}\right\}$ and $\left\{y_{i}^{n}\right\}$ by solving problem (34), (35) and problem (37), (38), respectively. Note that the conditional updates of $\left\{x_{i}^{n}\right\}$ and $\left\{y_{i}^{n}\right\}$ may either decrease or maintain but cannot increase the MSE. Monotonic convergence of this alternating algorithm follows directly from this observation. Finally, $\mathbf{F}_{0}^{n}$ and $\mathbf{G}_{0}^{n}$ can be obtained by substituting

$$
\lambda_{f, i}^{n}=\sqrt{\frac{y_{i}^{n}}{\left(\lambda_{s, i}^{n}\right)^{2} x_{i}^{n}+1}}, \quad \lambda_{g, i}^{n}=x_{i}^{n}, \quad \forall i, n
$$

back into (24) and (25), respectively. The optimal $\mathbf{B}^{n}$ is given by

$$
\mathbf{B}_{0}^{n}=\mathbf{V}_{s}^{n} \boldsymbol{\Lambda}_{b}^{n} \mathbf{V}_{b}^{n}
$$

where $\mathbf{V}_{b}^{n}$ is an $N_{b}^{n} \times N_{b}^{n}$ unitary matrix and

$$
\boldsymbol{\Lambda}_{b}^{n}=\left[\operatorname{diag}\left(\sqrt{\lambda_{g, 1}^{n}}, \sqrt{\lambda_{g, 2}^{n}}, \cdots, \sqrt{\lambda_{g, N_{b}^{n}}^{n}}\right), \mathbf{0}_{N_{b}^{n} \times\left(N_{s}-N_{b}^{n}\right)}\right]^{T} .
$$

Here $\operatorname{diag}(\cdot)$ denotes a diagonal matrix. Note that $N_{b}^{n}$ is automatically determined by the algorithm.

\section{Suboptimal Algorithm Using an Upper-Bound of MSE}

Useful insights can be obtained by studying an upper-bound of (31), which is given by

$$
\begin{aligned}
& \sum_{n=1}^{N_{c}} \sum_{i=1}^{M} \frac{a_{i}^{n} x_{i}^{n}+b_{i}^{n} y_{i}^{n}+2}{a_{i}^{n} b_{i}^{n} x_{i}^{n} y_{i}^{n}+a_{i}^{n} x_{i}^{n}+b_{i}^{n} y_{i}^{n}+1} \\
& \quad=\sum_{n=1}^{N_{c}} \sum_{i=1}^{M}\left(\frac{1}{a_{i}^{n} x_{i}^{n}+1}+\frac{1}{b_{i}^{n} y_{i}^{n}+1}\right) .
\end{aligned}
$$

Using (40) as the objective function, we develop a suboptimal algorithm by solving problem (40), (32), (33). We find that this optimization problem can be decomposed into the following two parallel subproblems. Subproblem 1 is written as

$$
\begin{aligned}
\min _{\left\{x_{i}^{n}\right\}} & \sum_{n=1}^{N_{c}} \sum_{i=1}^{M} \frac{1}{a_{i}^{n} x_{i}^{n}+1} \\
\text { s.t. } & \sum_{n=1}^{N_{c}} \sum_{i=1}^{M} x_{i}^{n} \leq p_{s}, \quad x_{i}^{n} \geq 0, \quad \forall i, n .
\end{aligned}
$$

While subproblem 2 is given by

$$
\begin{aligned}
\min _{\left\{y_{i}^{n}\right\}} & \sum_{n=1}^{N_{c}} \sum_{i=1}^{M} \frac{1}{b_{i}^{n} y_{i}^{n}+1} \\
\text { s.t. } & \sum_{n=1}^{N_{c}} \sum_{i=1}^{M} y_{i}^{n} \leq p_{r}, \quad y_{i}^{n} \geq 0, \quad \forall i, n .
\end{aligned}
$$

Interestingly, both problems optimize the MSE of the signal waveform estimation of single-hop parallel SISO Gaussian channels. In particular, problem (41), (42) optimizes the MSE of the source-relay channel, while problem (43), (44) optimizes that of the relay-destination channel. The solutions to problem (41), (42) and problem (43), (44) are given respectively by

$$
x_{i}^{n}=\frac{1}{a_{i}^{n}}\left[\sqrt{\frac{a_{i}^{n}}{\nu_{1}}}-1\right]^{\dagger}, \quad y_{i}^{n}=\frac{1}{b_{i}^{n}}\left[\sqrt{\frac{b_{i}^{n}}{\nu_{2}}}-1\right]^{\dagger}, \forall i, n
$$

where $\nu_{1}>0$ and $\nu_{2}>0$ are solutions to the following nonlinear equations, respectively.

$$
\begin{aligned}
& \sum_{n=1}^{N_{c}} \sum_{i=1}^{M} \frac{1}{a_{i}^{n}}\left[\sqrt{\frac{a_{i}^{n}}{\nu_{1}}}-1\right]^{\dagger}=p_{s} \\
& \sum_{n=1}^{N_{c}} \sum_{i=1}^{M} \frac{1}{b_{i}^{n}}\left[\sqrt{\frac{b_{i}^{n}}{\nu_{2}}}-1\right]^{\dagger}=p_{r} .
\end{aligned}
$$

Both equations can be solved by the bisection method [6].

Compared with the alternating algorithm (34), (35), (37), (38), the suboptimal algorithm (41)-(44) has a substantially reduced computational complexity, and thus, is very attractive in practice. Note that the upper-bound (40) is tight when the transmission power $p_{s}$ and $p_{r}$ are sufficiently high, since in such case, $a_{i}^{n} x_{i}^{n}+b_{i}^{n} y_{i}^{n} \gg 1$, and we have $a_{i}^{n} x_{i}^{n}+b_{i}^{n} y_{i}^{n}+$ $1 \approx a_{i}^{n} x_{i}^{n}+b_{i}^{n} y_{i}^{n}+2, \forall i, n$. In Section IV we will see that the suboptimal algorithm yields only a slight MSE increment compared with the alternating algorithm.

\section{Simulations}

In this section, we study the performance of the proposed non-regenerative multicarrier MIMO relay techniques through numerical simulations. For all examples, the channel between each transmit-receive antenna pair is modelled as the ETSI "Vehicular A" multipath channel environment [7]. An OFDM communication system with $N_{c}=64$ subcarriers is assumed. The channel matrices have zero-mean entries with variances 
$\sigma_{s}^{2}$ and $\sigma_{r}^{2}$ for $\mathbf{H}_{s}^{n}$ and $\mathbf{H}_{r}^{n}$, respectively. The signal-to-noise ratios (SNRs) are defined as

$$
\mathrm{SNR}_{\mathrm{S}} \triangleq \sigma_{s}^{2} p_{s} / N_{c}, \quad \mathrm{SNR}_{\mathrm{r}} \triangleq \sigma_{r}^{2} p_{r} / N_{c}
$$

for the source-relay and relay-destination links, respectively. The averaged MSE (AMSE) is computed as

$$
\operatorname{AMSE}=\frac{\sum_{q=1}^{N_{q}} \sum_{n=1}^{N_{c}} \operatorname{tr}\left(\left[\hat{\mathbf{s}}^{n}(q)-\mathbf{s}^{n}(q)\right]\left[\hat{\mathbf{s}}^{n}(q)-\mathbf{s}^{n}(q)\right]^{H}\right)}{N_{q} N_{c} N_{s}}
$$

where the average is carried out over $N_{q}=2000$ independent channel realizations.

We compare the proposed optimal algorithm, and the suboptimal algorithm with the following schemes.

- Relay-only suboptimal (ROS) algorithm: In this scheme, one chooses an identity source precoding matrix

$$
\mathbf{B}^{n}=\sqrt{\frac{p_{s}}{N_{s} N_{c}}} \mathbf{I}_{N_{s}}, \quad \forall n
$$

and $\mathbf{F}^{n}$ is given by (24) with $\boldsymbol{\Lambda}_{f}^{n}$ optimized for $\mathbf{B}^{n}$ in (46).

- Naive amplify-and-forward (NAF) algorithm: Here $\mathbf{B}^{n}$ is given by (46), while $\mathbf{F}^{n}$ is taken as

$$
\mathbf{F}^{n}=\sqrt{\frac{p_{r}}{N_{c} \operatorname{tr}\left(\mathbf{\Upsilon}^{n}\right)}} \mathbf{I}_{N_{r}}, \quad \forall n
$$

where $\mathbf{\Upsilon}^{n} \triangleq \frac{p_{s}}{N_{s} N_{c}} \mathbf{H}_{s}^{n}\left(\mathbf{H}_{s}^{n}\right)^{H}+\mathbf{I}_{N_{r}}, \quad \forall n$.

- Pseudo matched-filter (PMF) algorithm [8]: In this scheme, $\mathbf{B}^{n}$ is given by (46), and $\mathbf{F}^{n}$ is

$$
\mathbf{F}^{n}=\sqrt{\frac{p_{r}}{N_{c} \operatorname{tr}\left(\left(\mathbf{H}_{r}^{n}\right)^{H}\left(\mathbf{H}_{s}^{n}\right)^{H} \mathbf{\Upsilon}^{n} \mathbf{H}_{s}^{n} \mathbf{H}_{r}^{n}\right)}}\left(\mathbf{H}_{s}^{n} \mathbf{H}_{r}^{n}\right)^{H}, \quad \forall n .
$$

In our first example, we choose $N_{s}=N_{r}=N_{d}=3$, and $\mathrm{SNR}_{\mathrm{r}}=20 \mathrm{~dB}$. Fig. 1 shows the AMSEs of all algorithms versus $\mathrm{SNR}_{\mathrm{s}}$. It can be seen that the proposed optimal algorithm consistently yields the lowest AMSE over the whole $\mathrm{SNR}_{\mathrm{S}}$ range. The NAF and PMF algorithms have a high AMSE even at very high $\mathrm{SNR}_{\mathrm{s}}$. We also find that when all nodes have equal number of antennas, the ROS algorithm has a comparable performance to that of the optimal scheme.

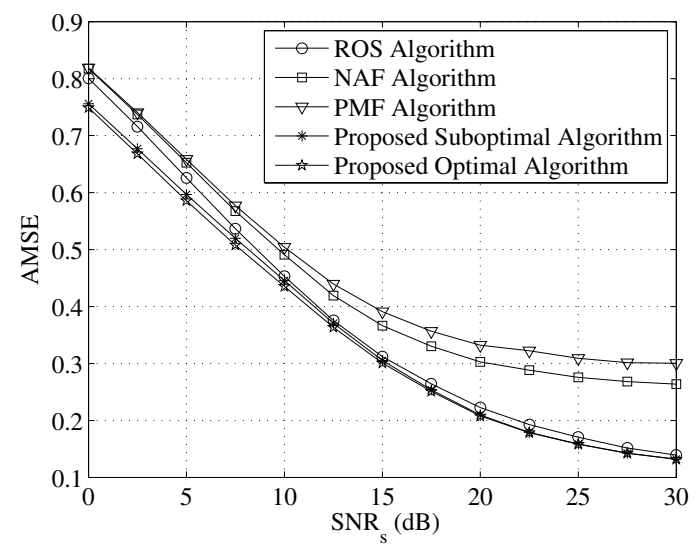

Fig. 1. AMSE versus $\mathrm{SNR}_{\mathrm{s}} . N_{s}=N_{r}=N_{d}=3, \mathrm{SNR}_{\mathrm{r}}=20 \mathrm{~dB}$.
In the second example, we set $N_{s}=5, N_{r}=6, N_{d}=4$, and fix $\mathrm{SNR}_{\mathrm{r}}$ to $20 \mathrm{~dB}$. The AMSEs of all algorithms except the PMF scheme versus $\mathrm{SNR}_{\mathrm{s}}$ are displayed in Fig. 2. Note that in contrast to other schemes, the PMF algorithm requires $N_{d}=N_{s}$. We find that the proposed optimal algorithm has the best performance. The performance of the ROS algorithm degrades significantly when all nodes have different number of antennas, because in this scheme, the source precoding matrix is "omnidirectional". From Figs. 1 and 2, we also find that the performance of the proposed upper-bound based suboptimal algorithm is very close to that of the proposed optimal alternating algorithm.

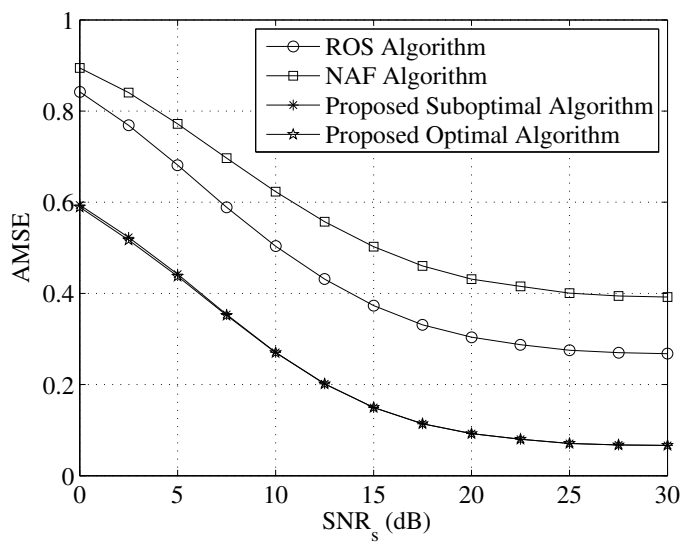

Fig. 2. AMSE versus $\mathrm{SNR}_{\mathrm{s}} . N_{s}=5, N_{r}=6, N_{d}=4, \mathrm{SNR}_{\mathrm{r}}=20 \mathrm{~dB}$.

\section{CONCLUSION}

We developed novel non-regenerative (linear precoding) techniques for multicarrier MIMO wireless relay communications. The proposed source and relay precoding matrices jointly convert the multicarrier MIMO relay channel into parallel SISO relay channels and minimize the MSE of the signal waveform estimation.

\section{REFERENCES}

[1] A. Sendonaris, E. Erkip, and B. Aazhang, "User cooperation diversity - part I and part II," IEEE Trans. Commun., vol. 51, pp. 1927-1948, Nov. 2003.

[2] J. N. Laneman, D. N. C. Tse, and G. W. Wornell, "Cooperative diversity in wireless networks: Efficient protocols and outage behavior," IEEE Trans. Inform. Theroy, vol. 50, pp. 3062-3080, Dec. 2004.

[3] X. Tang and Y. Hua, "Optimal design of non-regenerative MIMO wireless relays," IEEE Trans. Wireless Commun., vol. 6, pp. 1398-1407, Apr. 2007.

[4] I. Hammerström and A. Wittneben, "Joint power allocation for nonregenerative MIMO-OFDM relay links," in Proc. IEEE Intern. Conf. Acoustics, Speech, and Signal Processing, Toulouse, France, May 2006, vol. 4, pp. 49-52.

[5] A. W. Marshall and I. Olkin, Inequalities: Theory of Majorization and Its Applications, Academic Press, 1979.

[6] S. Boyd and L. Vandenberghe, Convex Optimization. Cambridge, U.K.: Cambridge University Press, 2004.

[7] "Universal Mobile Telecommunications System (UMTS); Selection Procedures for the Choice of Radio Transmission Technologies of the UMTS (UMTS 30.03 Version 3.2.0)," ETSI Standard, Document TR 101112 V3.2.0, 1998

[8] P. U. Sripathi and J. S. Lehnert, "A throughput scaling law for a class of wireless relay networks," in Proc. 38th Annual Asilomar Conference on Signals, Systems and Computers, Nov. 2004, vol. 2, pp. 1333-1337. 